\title{
Virtual Reality-Architecture-Neurosciences: Modeling and Evaluation of Immersive and Homeodynamic Hospital Environments at CAISM-UNICAMP
}

\author{
Keywords \\ Virtual Reality, Architecture-Neuroscience, Homeodynamic Environments and Products, \\ Pandemic and Post-Pandemic Contexts, CAISM-UNICAMP.
}

Studies have shown that the use of virtual reality devices (VR) as exposure therapy resources tend to produce favorable effects on the homeodynamic balance of patients with different diseases. The sensory and sensory-motor stimuli experienced through interaction with such technologies are able to promote, for instance, relief of pain intensity, stress levels reduction and reduced risks of hypertension syndrome in blood pressure tests. Thus, the emerging body of studies about the design, validation and development procedures of such ambiences is extremely fruitful and relevant, especially regarding their consistent contributions to the biological homeostasis of human beings. In this sense, this research aims to model humanized and homeodynamic hospital VR environments for nursing professionals of the Center for Integral Care to Women (CAISM-UNICAMP), capable of reducing their stress levels resulting from physical and mental overload, to which these professionals are daily exposed in the hospital settings, and further increased by the impacts of the COVID-19 pandemic. For this, the study starts from the mapping and identification of design parameters of humanized hospital environments, both through literature systematic review on transdisciplinary cooperation (e.g. between humanized hospital environments, intelligent biointerfaces and virtual reality), and data collected in field research (e.g. collection of architectural data, design, and observation of professionals' routine and semi-structured interviews with the healthcare team, before and after the experimental protocol application). The design and conception of these virtual environments are based on the cooperation between the fields of Architecture and Cognitive and Behavioral Neurosciences, especially subsidized by the concept of Homeodynamic Environments and Products. In addition, the investigation is also based on literature reviews about the use of VR in healthcare; a set of recommendations for conducting clinical studies using VR; exploration of VR soundscapes to reduce stress levels in hospitalized patients; and guidelines for conducting usability assessments for health-related VR applications for patient treatment, rehabilitation, and medical professional training. The tests and validation of the humanized and homeodynamic ambiences modeled are conducted with the nursing professionals of CAISM-UNICAMP, with the organization of participants in experimental and control groups. Such interventions are also associated and synchronized with the performance of intelligent biointerfaces, in order to collect consistent neuropsychophysiological data, performed during the immersion of the research subjects in the simulated environments. Thus, the results of this research aim to contribute to the reduction of stress levels of CAISM-UNICAMP nursing professionals, both in pandemic and postpandemic context. 